International Mathematical Forum, 1, 2006, no. 12, 597-602

\title{
THE MOL SOLUTION FOR THE ONE-DIMENSIONAL HEAT EQUATION SUBJECT TO NONLOCAL CONDITIONS
}

\author{
M. Javidi \\ Department of Mathematics, Razi University \\ Kermanshah 67149, Iran
}

\begin{abstract}
In this paper,the problem of solving the one-dimensional equation $\frac{\partial \varphi}{\partial t}-\frac{\partial^{2} \varphi}{\partial x^{2}}=g(x, t)$ subject to given initial and nonlocal conditions by the method of lines(MOL). Firstly theory of application of MOL on heat equation presented. Secondly we considers some examples to show goodness of new method. Computational results using a code based on our method are presented for this examples.
\end{abstract}

Mathematics Subject Clasification: 35K05, 65N06, 65N40, 65N22

Keywords: Heat equation, nonlocal conditions, MF-BDF and MF-MEBDF method, MOL

\section{Introduction}

A problem which arises in the quasi-static theory of thermoelasticity (Day[10],[9]) requires solving the one-dimensional heat equation

$$
\frac{\partial \varphi}{\partial t}-\frac{\partial^{2} \varphi}{\partial x^{2}}=g(x, t) \quad \text { for } \quad x \in(0,1) \text { and } t>0
$$

subject to the initial condition

$$
\varphi(x, 0)=u(x) \text { for } \quad x \in[0,1]
$$

and the nonlocal conditions

$$
\left.\begin{array}{l}
\varphi(0, t)=\int_{0}^{1} p(x) \varphi(x, t) d x+r(t) \\
\varphi(1, t)=\int_{0}^{1} q(x) \varphi(x, t) d x+v(t)
\end{array}\right\} \quad \text { for } \quad t>0
$$


where $x$ and $t$ are the spatial and time coordinates respectively, $\varphi$ is the unknown function of $x$ and $t$ be determined and $g, u, p, q, r$ and $v$ are suitably prescribed function.

Investigators like Ang [11] had studied the problem and introduced the Laplace transform domain methods solving it numerically. An approach related to finite difference method, which has found interesting applications in the numerical treatment of partial differential equations, is the method of lines. It makes use of the finite difference method in the construction of discreteanalytical model of a given partial differential equation in which all but one of its independent variables discretized. This procedure leads to a system of ordinary differential equations in the remaining variable.

\section{Semi discrete models for heat equation}

To solve (1)-(3), the problem is discretized in respect to $x$ by finite difference method. For this we define a uniform partition $P$ as:

$$
P: a=x_{0}<x_{1}<\cdots<x_{k}<x_{k+1}<\cdots<x_{2 n+1}=b,
$$

which a constant spacing $h=x_{k+1}-x_{k}, k=1,2, \cdots, 2 n$.

We use an $O\left(h^{4}\right)$ central difference scheme to estimate $\frac{\partial^{2} \varphi}{\partial x^{2}}$ on internal nods. Hence we have the following difference scheme :

for $x=x_{1}$

$$
\left.\frac{\partial^{2} \varphi}{\partial x^{2}}\right|_{x=x_{1}} \cong \frac{1}{12 h^{2}}\left(45 y_{1}-154 y_{2}+214 y_{3}-156 y_{4}+61 y_{5}-10 y_{6}\right)=E_{1}(t),
$$

for $x=x_{2}$

$$
\left.\frac{\partial^{2} \varphi}{\partial x^{2}}\right|_{x=x_{2}} \cong \frac{1}{24 h^{2}}\left(20 y_{1}-30 y_{2}-8 y_{3}+28 y_{4}-12 y_{5}+2 y_{6}\right)=E_{2}(t),
$$

for $x=x_{i}$

$$
\left.\frac{\partial^{2} \varphi}{\partial x^{2}}\right|_{x=x_{i}} \cong \frac{1}{12 h^{2}}\left(-2 y_{i-2}+32 y_{i-1}-60 y_{i}+32 y_{i+1}-2 y_{i+2}=E_{i}(t),\right.
$$

for $x=x_{2 n}$

$\left.\frac{\partial^{2} \varphi}{\partial x^{2}}\right|_{x=x_{2 n}} \cong \frac{1}{24 h^{2}}\left(20 y_{2 n+1}-30 y_{2 n}-8 y_{2 n-1}+28 y_{2 n-2}-12 y_{2 n-3}+2 y_{2 n-4}\right)=E_{2 n}(t)$,

for $x=x_{2 n+1}$

$\left.\frac{\partial^{2} \varphi}{\partial x^{2}}\right|_{x=x_{2 n+1}} \cong \frac{1}{12 h^{2}}\left(45 y_{2 n+1}-154 y_{2 n}+214 y_{2 n-1}-156 y_{2 n-2}+61 y_{2 n-3}-10 y_{2 n-4}\right)$ 


$$
=E_{2 n+1}(t)
$$

where $y_{k}=y_{k}(t) \cong \varphi\left(x_{k}, t\right) \quad, \quad k=1,2, \cdots, 2 n$.

By (5)-(9) the following system of ordinary differential equations is obtained :

$$
y_{i}^{\prime}(t)=E_{i}(t)+g_{i}(t) \quad, \quad k=1,2, \cdots, 2 n
$$

where $g_{i}(t)=g_{i}=g\left(x_{i}, t\right)$ whit following condition:

$$
y_{i}(0)=\varphi\left(x_{i}, 0\right)=u\left(x_{i}\right)=u_{i} .
$$

If we use the simpson rule to conditions (3) respect to $x_{i}$ by definition of partition $P$ as (4) we have:

$$
\begin{aligned}
& \varphi(0, t) \cong \frac{h}{3}\left[p\left(x_{1}\right) \varphi\left(x_{1}, t\right)+4 p\left(x_{2}\right) \varphi\left(x_{2}, t\right)+\cdots\right. \\
& \left.+4 p\left(x_{2 n}\right) \varphi\left(x_{2 n}, t\right)+p\left(x_{2 n+1}\right) \varphi\left(x_{2 n+1}, t\right)\right]+r(t)
\end{aligned}
$$

and

$$
\begin{aligned}
& \varphi(1, t) \cong \frac{h}{3}\left[q\left(x_{1}\right) \varphi\left(x_{1}, t\right)+4 q\left(x_{2}\right) \varphi\left(x_{2}, t\right)+\cdots\right. \\
& \left.+4 q\left(x_{2 n}\right) \varphi\left(x_{2 n}, t\right)+q\left(x_{2 n+1}\right) \varphi\left(x_{2 n+1}, t\right)\right]+v(t) .
\end{aligned}
$$

We solve the (12)-(13) respect to $y_{1}$ and $y_{2 n+1}$ as following:

$$
\begin{aligned}
& y_{2 n+1}=\frac{1}{A}\left[\frac{h^{2} q_{1}}{9-3 h p_{1}}\left(4 p_{2} y_{2}+\cdots+4 p_{2 n} y_{2 n}+\frac{3 r(t)}{3-h p_{1}}\right)\right. \\
& \left.+\frac{h}{3}\left(4 q_{2} y_{2}+\cdots+4 q_{2 n} y_{2 n}\right)\right]=\frac{B}{A} \\
& y_{1}=\frac{h}{3-h p_{1}}\left(4 p_{2} y_{2}+\cdots+4 p_{2 n} y_{2 n}+p_{2 n+1} \times \frac{B}{A}\right)+\frac{3 r(t)}{3-h p_{1}},
\end{aligned}
$$

where $p_{i}=p\left(x_{i}\right), q_{i}=q\left(x_{i}\right) \quad, \quad i=1,2, \cdots, 2 n$ and

$$
\begin{gathered}
A=1-\frac{q_{1} h^{2} p_{2 n+1}}{9-3 h p_{1}}-\frac{h q_{2 n+1}}{3}, \\
B=\frac{h^{2} q_{1}}{9-3 h p_{1}}\left(4 p_{2} y_{2}+\cdots+4 p_{2 n} y_{2 n}+\frac{3 r(t)}{3-h p_{1}}\right)+\frac{h}{3}\left(4 q_{2} y_{2}+\cdots+4 q_{2 n} y_{2 n}\right),
\end{gathered}
$$

Then we can combine the relations $(10),(14),(15)$ we have the following system of algebric differential equations:

$$
\left\{\begin{array}{c}
y^{\prime}(t)=f(t, y) \\
y(0)=y_{0}
\end{array}\right.
$$

Consider system (16) and suppose that it is stiff. In the backward differentiation formula (BDF), we have

$$
y_{n}=\sum_{j=1}^{k} \alpha_{j} y_{n-j}+h \beta_{k} f\left(t_{n}, y_{n}\right),
$$


where $k$ is the order of scheme and the values of $\alpha_{j}$ and $\beta_{k}$ are given in[10]. From (17) we have the following system of nonlinear equations to be solved:

$$
F\left(x_{n}\right)=x_{n}-h f\left(t_{n}, \alpha_{n}+\beta_{k} x_{n}\right)
$$

Where $\alpha_{n}=\sum_{j=1}^{k} \alpha_{j} y_{n-j}$ and $x_{n}=h f\left(t_{n}, y_{n}\right)$, see[4].Usually, to solve a system like (18), a modified Newton method is used. Then a direct method is usually used to solve any resulting system of linear equations. Hence in each step, we need to obtain the jacobian matrix and its related decomposed matrices. Therefore causing more computational and running time. In matrix free (MF-BDF) method, the jacobian matrix is not used explicitly, because the exact Newton method is used and then the IOM algorithm,see[12], is applied to solve the resulting system of linear equations. Cash, modified the BDF method, this modification led to the EBDF method[3], and MEBDF method [2] . In [7], authors applied the Cashes modification to the MF-BDF scheme and obtained a new method to solve ODE's and PDE's. They called their method MF-MEBDF.

In this study we want to apply the MF-BDF and MF-MEBDF methods solve system of DAE's.

\section{$3 \quad$ Numerical examples}

In this section, we apply the method of solution described above to solve the problem defined by (1)-(3) for particular $g, u, p, q, r$ and $v$.

Example 1.The first problem is selected from[11] . In this example, we take

$$
\begin{gathered}
g(x, t)=-x(x-1) \exp (-t) \\
u(x)=x(x-1)-2 \\
p(x)=\frac{12}{13}, \quad q(x)=\frac{12}{13} \\
r(t)=0, \quad v(t)=0 .
\end{gathered}
$$

The analytical solution of the problem is

$$
\varphi(x, t)=[x(x-1)-2] \exp (-t) .
$$

Numerical results by 21 line is presented in Table 1. 


\begin{tabular}{|c|c|c|c|c|}
\hline & $M F-M E B D F$ & $M F-M E B D F$ & $M F-B D F$ & $M F-B D F$ \\
\hline$t$ & MaxErr & MinErr & MaxErr & MinErr \\
\hline 0.2 & $1.45 E-10$ & $1.29 E-10$ & $2.34 E-9$ & $1.25 E-9$ \\
\hline 0.4 & $1.50 E-5$ & $1.37 E-5$ & $7.67 E-4$ & $5.89 E-4$ \\
\hline 0.6 & $1.23 E-5$ & $1.12 E-5$ & $6.58 E-4$ & $2.34 E-4$ \\
\hline 0.8 & $3.65 E-10$ & $3.27 E-10$ & $5.78 E-9$ & $3.05 E-9$ \\
\hline 1.0 & $2.73 E-10$ & $2.54 E-10$ & $4.35 E-9$ & $2.15 E-9$ \\
\hline
\end{tabular}

Table1

Example 2.The second problem is selected from[11]. In this example, we take

$$
\begin{gathered}
g(x, t)=\frac{-2\left(x^{2}+t+1\right)}{(t+1)^{3}} \\
u(x)=x^{2} \\
p(x)=x \quad, \quad q(x)=x \\
r(t)=-\frac{1}{4(t+1)^{2}} \quad, \quad v(t)=\frac{3}{4(t+1)^{2}} .
\end{gathered}
$$

The analytical solution of the problem is

$$
\varphi(x, t)=\left(\frac{x}{t+1}\right)^{2} .
$$

Numerical results by 21 line is presented in Table 2 .

\begin{tabular}{|c|c|c|c|c|}
\hline & $M F-M E B D F$ & $M F-M E B D F$ & $M F-B D F$ & $M F-B D F$ \\
\hline$t$ & MaxErr & MinErr & MaxErr & MinErr \\
\hline 0.2 & $2.99 E-10$ & $1.45 E-11$ & $4.32 E-9$ & $3.25 E-10$ \\
\hline 0.4 & $6.57 E-5$ & $1.82 E-7$ & $4.35 E-5$ & $1.99 E-6$ \\
\hline 0.6 & $4.43 E-5$ & $1.22 E-7$ & $8.97 E-5$ & $2.88 E-6$ \\
\hline 0.8 & $6.88 E-10$ & $5.91 E-12$ & $1.23 E-9$ & $3.55 E-10$ \\
\hline 1.0 & $1.56 E-9$ & $1.48 E-11$ & $2.17 E-8$ & $8.99 E-9$ \\
\hline
\end{tabular}

Table 2

where

$$
\begin{gathered}
\text { MaxErr }=\max \left|y_{k}(t)-\varphi\left(x_{k}, t\right)\right|, \quad k=1,2, \cdots, 2 n \\
\text { MinErr }=\min \left|y_{k}(t)-\varphi\left(x_{k}, t\right)\right| \quad, \quad k=1,2, \cdots, 2 n
\end{gathered}
$$




\section{References}

[1] J. R. Cash,and Psihoyios., The MOL solution of time dependent partial differentail equations ,Comp. Math. Applic., 31.(1996),no.,69-78.

[2] J. R. Cash,The integration of stiff initial value problems in ODE's using modified extended backward formula. ,Comp. Math. Applic.,9(1983),645657.

[3] J. R.Cash, On the integration of stiff system of ODE's using extended backward formula.Numer. Math, 34(1980),no.2,235-246.

[4] J. R.Cash, The numerical solution of ordinary differential equations, Proc. Comp. Phil. Soc, 76 (1974), 443-457.

[5] M. Berzins, and P.M. Dew, A note on $\mathrm{C}^{0}$ Chebyshev methods for parabolic PDE's,IMA, J, Numer, Anal, 17(1987) 15-37.

[6] M. T.Darvishi, and M.Javidi, Method of lines for solving system of time dependent partial differential equations, WSEAS Transations on Mathematics, 1(2002),no.1,218-222.

[7] S. M.Hosseini, and G.Hojatti, Matrix free MEBDF method for the solution of stiff systems of ODE's, Mathematical and computer Modeling ,29 (1999), $67-77$.

[8] W. T. Ang, A crack in an anisotropic layered material under the action of impact loading, ASME J, Appl , 55 (1988)122-125.

[9] W. A. Day, Adecreasing property of solutions of parabolic equations with applications to thermoelasticity ,Quart. Appl.Math ,41 (1983)468-475.

[10] W. A. Day , Extention othe heat equation to linear thermoelasticity and other theores. Quart. Appl.Math, 40(1982),319-330.

[11] W. T.Ang,A method of solution for the one-dimensional heat equation subject to nonlocal conditions, Southeast asian bulletin of mathematics. , 26(2002), 185-191.

[12] Y.Saad, Krylove subspace methods for solving large unsymmetric linear systems, Math. Comp, 76(1974), 443-457.

\section{Received: September 5, 2005}

